\title{
28 Research Square Effect of Air Pollution on Age at Menarche
}

Iwona Wronka ( $\Delta$ iwona.wronka@uj.edu.pl)

Jagiellonian University

Katarzyna Kliś

University of Wroclaw

\section{Research Article}

Keywords: Air Pollution, Menarche, sulphur dioxide, childhood

Posted Date: November 19th, 2021

DOI: https://doi.org/10.21203/rs.3.rs-1057716/v1

License: (c) (1) This work is licensed under a Creative Commons Attribution 4.0 International License. Read Full License 


\section{Abstract \\ Objectives}

to analyse the association between the degree of air pollution (suspended particulate matter, sulphur dioxide, benzopyrene levels) in the location of residence during childhood and adolescence and the age at menarche

\section{Methods}

Anthropometric measurements were performed, and questionnaire data were collected from 1,257 women, aged 19-25 years

\section{Results}

Negative relationships between age at menarche and suspended particulate matter as well as nitrogen levels were found. A similar trend was observed in an analysis of the relationship between age at menarche and the complex air pollution index. The tendency for age at menarche to decrease together with worsening air quality was also visible after adjusted for socioeconomic status. Girls exposed to high suspended particulate matter levels were characterised by higher risk of early age at menarche.

\section{Conclusion}

High levels of air pollution are related to younger age at menarche and the risk of the menstruation onset below $11 \mathrm{y}$.

\section{Introduction}

Ambient air quality is considered one of key determinants of human health [1-2]. Since biological development reflects an individual's condition and is deeply affected both by chronic and frequent shortterm illnesses, we may expect those differences due to ambient air quality will translate into variations in the rate of growth and maturation. In addition, air pollutants contain endocrine active substances which may impact human development and lowering the age at first menstruation in girls [3]. To date, however, those issues were analyzed in only a small number of published studies [4-14]. This is still a topical and important research issue, as many children from different countries are growing up in a poor air quality environment.

Menarche is an important milestone in human biological development and is often considered a fertility signal. Girls experience menarche at different ages but usually it occurs between the ages of 10 and 16 years. Besides genetics, menarche is also influenced by socioeconomic and environmental factors [2]. 
Numerous studies analyse factors modifying the age at menarche, however, data on the impact of environmental pollution on the onset of menstruation is limited. To the best of our knowledge, such research has not yet been carried out in any European country.

It seems reasonable to investigate this problem, as many studies have shown a significant association between age at menarche and health status in adulthood. Late menarche is associated with osteoporosis and increased fracture risk [15]. The early onset of menstruation increases the risk of obesity and numerous conditions such as breast and ovarian cancer, the metabolic syndrome, cardiovascular diseases and hypertension [16-19]. Additionally, numerous studies carried out over the past 150 years have revealed a tendency for a decreasing age at menarche. Certain researchers state that the consequence of this phenomenon is the rise in the incidence of lifestyle diseases and the obesity epidemic [20-21].

The aim of the study was to analyze the association between the degree of air pollution (suspended particulate matter, sulphur dioxide, benzopyrene levels) in the location of residence during childhood and adolescence and the age at menarche.

\section{Material And Methods}

The research was carried out in the period from 2015 to 2017 among 1257 female university students aged 19 to 25 years. The study protocol was approved by the Bioethics Committee of the Jagiellonian University (opinion no.122.612047.2016). Data were collected following the ethical principles as stated in the Declaration of Helsinki. Informed consent was obtained from all individual participants included in the study.

Age at menarche was assessed based on the date provided by the subjects. Students were asked to provide the date of their first menstruation with the accuracy within one month or a quarter. The age at menarche was calculated from the date of menarche and the date of birth. In cases when, instead of an exact date, participants reported only month and year of menarche, the 15th day of an indicated month was used for calculation, and when the date of menarche was reported approximately in the range of 2-3 months, the midpoint of the reported period was used for calculation.

The level of each analyzed environmental pollution: particular mater $\left(\mathrm{PM}_{10}, \mathrm{PM}_{2.5}\right)$, sulphur dioxide $\left(\mathrm{SO}_{2}\right)$, nitric oxide (NO) and benzene $\left(\mathrm{C}_{6} \mathrm{H}_{6}\right)$ in the town of residence during childhood and adolescence was be determined on the basis of the data made available by the Chief Inspectorate for Environmental Protection. Previous place of residence was divided into 3 categories, taking into account the air quality class to which the area was classified for most of the time (at least within 10 years). Class 1 comprised zones where annual pollutant values and the number of days per year with exceedances were below the allowable limit; Class 2 - zones with annual values below the permissible limit, but with the number of days of exceeding the norm above the limit, Class 3 included zones above the limit. Based on all the level of each pollutant a complex air pollution index was established. To assess the joint effect of air 
pollutants, principal component analyses were used to group pollutants (PM10, PM2.5 SO2, NO, and $\mathrm{C} 6 \mathrm{H} 6)$ to represent a source-related mixture. Since the first component was relatively high, scores for the single component were used as an indicator of air quality. Tertiles of the component were used to identify three air quality classes: good, moderate or unhealthy.

Socio-economic status was included in the analysis as covariates and it was determined on the basis of variables considered reliable indicators of living conditions and lifestyle in Poland and Europe: degree of urbanization of the place of residence, father's and mother's education, number of siblings, and financial situation. The following categories were created: for the place of residence in childhood and adolescence: village, city up to 100,000 inhabitants, city above 100,000 inhabitants; for mother's and father's education: vocational, secondary, higher; for number of siblings: none, one, two, three or more. Financial situation during childhood and adolescence was assessed based upon subject's responses to a survey question: 'Do you consider the economic situation in your home as: below average and bad, average, good, very good, changeable and/or hard to specify'. As there was only one response in the last category, it has been omitted. Based on all the above data, a SES evaluation index was established and subjects were qualified as belonging to families of low, average, or high SES. The division was introduced on the basis of the value of the first component obtained in the principal components analysis (PCA).

The study design is fully described elsewhere [5].

\section{Statistical Methods}

Statistical analyses were performed with the use of statistical software Statistica 13.0 (by StatSoft Polska). This manuscript is a continuation of our research analyzing the impact of air pollution on biological development therefore, we used the same statistical methods as previously [5]. The ShapiroWilk test was applied for stature distribution normality assessment, and the Levene's test for assessing the equality of variance of the analysed data. Air pollutions were analysed as categorical variables. In statistical analysis the generalized linear model (GLM) was applied. Analyses were conducted in three steps. Model 1 included the level of each analysed air pollutant: particulate matter (PM10, PM2.5), sulphur dioxide (SO2), nitric oxide (NO) and benzene ( $\mathrm{C} 6 \mathrm{H} 6)$. Model 2 was adjusted for the degree of urbanization of the place of residence. In case of subject who moved during childhood or adolescence, the degree of urban development was calculated as the average category of all residences of the subject, weighted by the length of each residential period. Model 3 included as covariates all socio-economic variables: degree of urbanization of the place of residence, father's and mother's education, number of siblings, and financial situation. All adjustment covariates were selected a priori [5].

To assess the effect of multiple-pollutant exposure on the age at menarche two-way analysis of variance and logistic regression were applied. Significance in all statistical tests was set at the level of at least $\mathrm{p}<0.05[5]$. 


\section{Results}

\section{Effects of air pollution on age at menarche}

With regard to single pollutants, significant differences in mean age at menarche were reported depending on suspended particulate matter and nitrogen levels. Mean age at menarche dropped as the levels of the pollutants increased (Table 1).

Table 1

Age at menarche (mean \pm standard deviation) in relation to air pollutants levels in the place of residence during childhood and adolescence

\begin{tabular}{|c|c|c|c|c|c|c|}
\hline & \multicolumn{3}{|c|}{ Pollutants level } & \multicolumn{3}{|l|}{ p-values } \\
\hline & Class 1 & Class 2 & Class3 & Model 1 & Model & Model \\
\hline & Low & Medium & High & & 2 & 3 \\
\hline $\mathrm{PM}_{10}$ & $13.32 \pm 1.45$ & $12.68 \pm 1.34$ & $12.54 \pm 1.28$ & 0.0000 & 0.0000 & 0.0003 \\
\hline $\mathrm{PM}_{2.5}$ & $13.18 \pm 1.39$ & $12.79 \pm 1.45$ & $12.55 \pm 1.17$ & 0.0090 & 0.0097 & 0.0089 \\
\hline benzene & $12.61 \pm 1.38$ & $12.91 \pm 1.34$ & $12.73 \pm 1.30$ & 0.1469 & 0.1520 & 0.1422 \\
\hline $\mathrm{SO}_{2}$ & $12.82 \pm 1.37$ & $12.75 \pm 1.38$ & $12.71 \pm 1.30$ & 0.3800 & 0.4201 & 0.3946 \\
\hline NO & $12.98 \pm 1.37$ & $12.71 \pm 1.35$ & $12.62 \pm 1.33$ & 0.0031 & 0.0026 & 0.0034 \\
\hline Whole models & & & & 0.0000 & 0.0002 & 0.0001 \\
\hline
\end{tabular}

A similar trend was observed in an analysis of the relationship between age at menarche and the complex air pollution index (Table 2). Post-hoc test results (Tuckey's HSD) demonstrated significant differences both between the group from areas of good and unhealthy air quality $(p=0.0000)$ and between the group from areas of moderate and groups from areas of good $(p=0.0083)$ and unhealthy air quality $(p=0.0345)$. The tendency for age at menarche to decrease together with worsening air quality was also visible after subjects were divided into groups of various SES (Table 2). 
Table 2

Age at menarche $t$ in relation to air quality and socioeconomic status (SES)

\begin{tabular}{|c|c|c|c|c|c|c|}
\hline SES & Air quality & $\mathbf{N}$ & Mean & sd & $\begin{array}{l}\text { Result } \\
\text { of MANOVA }\end{array}$ & Signifacantly differed groups \\
\hline low & & 414 & 12.83 & 1.31 & Main effects & \\
\hline average & & 423 & 12.79 & 1.25 & $F=0.43$ & \\
\hline \multirow[t]{4}{*}{ good } & & 420 & 12.87 & 1.34 & $p=0.6521$ & \\
\hline & 1. good & 361 & 13.07 & 1.34 & Main effects & $1-2$ \\
\hline & 2. moderate & 450 & 12.85 & 1.29 & $F=1.519$ & $1-3$ \\
\hline & 3. unhealthy & 446 & 12.58 & 1.16 & $p=0.0000$ & $2-3$ \\
\hline \multirow[t]{3}{*}{ low } & 1. good & 126 & 13.11 & 1.33 & Interactions & $1-2$ \\
\hline & 2. moderate & 150 & 12.75 & 1.21 & $F=0.78$ & $1-3$ \\
\hline & 3. unhealthy & 138 & 12.62 & 1.28 & $p=0.5391$ & \\
\hline \multirow[t]{3}{*}{ average } & 1. good & 128 & 12.94 & 1.29 & & \\
\hline & 2. moderate & 144 & 12.89 & 1.25 & & $1-3$ \\
\hline & 3. unhealthy & 151 & 12.55 & 1.22 & & $2-3$ \\
\hline \multirow[t]{3}{*}{ good } & 1. good & 107 & 13.17 & 1.24 & & \\
\hline & 2. moderate & 156 & 12.91 & 1.27 & & $1-3$ \\
\hline & 3. unhealthy & 157 & 12.55 & 1.29 & & $2-3$ \\
\hline
\end{tabular}

\section{Effects of air pollution on the risk of early menarche}

A logistic regression model was applied in the assessment of the risk of early first menstruation (age below 11) depending on air pollution levels (Table 3 ). Girls exposed to high suspended particulate matter levels, both $\mathrm{PM}_{10}(\mathrm{p}=0.0182)$ and $\mathrm{PM}_{2.5}(\mathrm{p}=0.01043)$ were characterized by higher risk of early age at menarche. OR values were calculated as $3.18(95 \% \mathrm{Cl} 2.29-4.69)$ and 3.25 (95\% $\mathrm{Cl}$ 2.34-4.80) respectively, which suggests that the likelihood of accelerated age at menarche in the group living in areas with high levels of particulate matter was more than three times higher than in the group from areas with low levels of particulate matter (OR $=1$, Ref). 
Table 3

The risk of the onset of menstruation below $11 \mathrm{y}$. in relation to the air pollution

\begin{tabular}{|c|c|c|c|c|}
\hline \multicolumn{2}{|c|}{ Pollutants level } & OR & $95 \% \mathrm{Cl}$ & p-values \\
\hline \multirow{3}{*}{$\mathrm{PM}_{10}$} & low & \multicolumn{3}{|c|}{1 (Ref.) } \\
\hline & medium & 1.92 & $1.61-2.07$ & \\
\hline & high & 3.18 & $2.29-4.69$ & 0.0182 \\
\hline \multirow[t]{3}{*}{$\mathrm{PM}_{2.5}$} & low & \multicolumn{3}{|c|}{1 (Ref.) } \\
\hline & medium & 2.01 & $1.73-2.16$ & \\
\hline & high & 3.25 & $2.34-4.80$ & 0.01043 \\
\hline \multirow[t]{3}{*}{ benzene } & low & \multicolumn{3}{|c|}{1 (Ref) } \\
\hline & medium & 0.98 & $0.61-1.28$ & \\
\hline & high & 1.11 & $0.90-1.64$ & 0.1568 \\
\hline \multirow[t]{3}{*}{$\mathrm{SO}_{2}$} & low & \multicolumn{3}{|c|}{1 (Ref.) } \\
\hline & medium & 1.03 & $0.78-1.38$ & \\
\hline & high & 1.22 & $1.01-2.14$ & 0.1725 \\
\hline \multirow[t]{3}{*}{ NO } & low & \multicolumn{3}{|c|}{1 (Ref.) } \\
\hline & medium & 1.06 & $0.55-1.22$ & \\
\hline & high & 1.47 & $0.65-1.35$ & 0.0844 \\
\hline
\end{tabular}

\section{Discussion}

On the basis of our study, we may conclude that air pollution is a significant modifier of the rate of development. Our results revealed that accelerated first menstruation occurs in girls who in early childhood and adolescence lived in areas of poor air quality and high suspended particulate matter levels. To the best of our knowledge, only one previous study analyzed the effect of pollution on age at menarche, reporting similar results. In Korean girls heightened $\mathrm{PM}_{10}$ level was associated with earlier age at menarche [22]. The literature of the subject also includes studies on other maturation indicators. McGuin et al. analyzed the effect of traffic pollutants and found that girls living within $150 \mathrm{~m}$ from a main road, or a highway reached pubarche a younger age. No relationship between traffic pollutants and thelarche was found [9]. Meanwhile, research by Huang et al. demonstrated that exposure to $\mathrm{PM}_{10}$ delayed thelarche [8]. 
Several studies analyzed the influence of lead, which may be contained in suspended particulate. It was found that, unlike particulate, increased lead level in blood is related to later age at menarche [13-14].

The biological mechanism in which suspended particulate affects age at first menstruation is still unknown. What we do know, however, is that those pollutants may induce oxidative stress, causing inflammations in respiratory and cardiovascular system [23]. Moreover, they may contain endocrine active substances, which affect e.g., nervous, endocrine or reproductive systems [24-25]. Endocrine disrupters may trigger the secretion of kisspeptin and initiate the secretion of gonadotropin releasing hormone, thereby promoting hypothalamic maturation and consequently earlier puberty [26-27]. It was also proved that some compounds found in particle matter, especially polycyclic aromatic hydrocarbons which mimic estrogens, may cause the disturbances in hormonal function of the female reproductive system [28-30].

It should be noted that the composition of suspended particulate matter may vary depending on location and that other airborne substances may exacerbate the adverse effect of particulate on the body. For this reason, further research on the subject is recommended, preferably in groups living in various geographic environments. Poland is a country with poor air quality, where pollution is generated mostly by burning coal and solid fuels in stoves. Smog forms usually in winter in frosty weather associated with high pressure systems. It is characterized by high concentration of particulate matter, especially $\mathrm{PM}_{10}$ and $\mathrm{PM}_{2.5}$, as well as benzopyrene, with no increase in sulphur dioxide concentrations.

Our study shows that high levels of air pollution are related to younger age at menarche and the risk of the menstruation onset below $11 \mathrm{y}$. This finding may be helpful when providing an explanation of the relationship between age at menarche and health conditions in adulthood, e.g., conditions of respiratory, cardiovascular, hormonal and reproductive system. They also may prove helpful in disease prevention. It seems that one of the key elements in this area is to draw attention to ensuring appropriate conditions for biological development in childhood and eliminating factors which may accelerate or decelerate maturation and, as a consequence, affect biological condition of an individual in her adulthood.

The main limitation of our study is potential misclassification of exposure. The assessment of exposure to airborne pollutants was based on data averaged over the period of more than a decade for a given city/town/village. Obviously, such evaluation is far from precise and constitutes a limitation of the study. Children are not exposed to pollutants only in their place of residence, but also in their school, during their travel to school and all other activities, such as recreational activities, etc. However, it is not possible to precisely determine exposure to harmful substances in the air for large groups and long periods of exposure. For this reason, records of air pollution from stations monitoring the nearest residential addresses are often used in epidemiological studies [31-33].

\section{Conclusions}


The air pollutants $\mathrm{PM}_{10}, \mathrm{PM}_{2.5}$, and $\mathrm{NO}$ assessed separately, negatively affect the age at menarche, also after standardization to confounders, such as the level of urbanization of dwelling place or other socioeconomic factors (parents' education, number of siblings and financial conditions). Additionally, high levels of air pollution were related to the risk of the menstruation onset below $11 \mathrm{y}$.

The significant differences in the age at menarche age were found depending on the air quality index, which reflects the combined effect of all pollutants assessed. Girls growing up in an area with unhealthy air quality had the onset of menstruation at a younger age than girls from areas with good and moderate air quality.

Our findings confirmed that air pollution is a significant endocrine disrupting factor. Negative effects of elevated levels of air pollutants can be seen as early as adolescence. Given the correlation between early menarche and the risk of various diseases, it can be expected that air pollution in the place of residence during childhood may also have long term negative health effects.

\section{Declarations}

\section{Author Contributions:}

Conceptualization and Methodology- K.K.; Data Collecting- K.K. and I.W; Statistical Analysis - K.K.; Original Draft Preparation - K.K.; Review and Editing - K.K. and I.W.; Supervision - I.W.

\section{Funding:}

This research received no specific grant from any funding agency, commercial entity or not-for-profit organization.

\section{Disclosure:}

The authors report no conflict of interest.

\section{Declaration of competing financial interests}

The authors declare they have no actual or potential competing financial interests.

\section{References}

1. Kelly, F. J. \& Fussell, J. C. Air pollution and airway disease. Clin. Exp. Allergy, 41 (8), 1059-107 (2011).

2. Kampa, M. \& Castanas, E. Human health effects of air pollution. Environ. Pollut, 151 (2), 362-367 (2008).

3. Karapanou, O. \& Papadimitriou, A. Determinants of menarche. Reprod. Biol Endocrinol, 8, 115 (2010). 
4. Salgueiro-Gonzalez, N., Lopez de Alda, M., Muniategui-Lorenzo, S. \& Prada-Rodrígueza, D. Barcelóbc D. Determination of 13 estrogenic endocrine disrupting compounds in atmospheric particulate matter by pressurised liquid extraction and liquid chromatography-tandem mass spectrometry. Analyt. Bioanaly.t Chem, 405 (27), 8913-8923 (2013).

5. Klis, K. \& Wronka, I. Associations between childhood and adolescence exposure to air pollution and adult height in polish women. Environ. Res, 189, 109965 (2020).

6. Parasin, N., Amnuaylojaroen, T. \& Saokaew, S. Effect of air pollution on obesity in children, A systematic review and meta-analysis. Children, 8 (5), 327 (2021).

7. Huang, J. V., Leung, G. M. \& Schooling, C. M. The association of air pollution with height, Evidence from Hong Kong's “Children of 1997” birth cohort. Am. J. Hum. Biol, 30, e23067 (2018).

8. Huang, J. V., Leung, G. M. \& Schooling, C. M. The association of air pollution with pubertal development, Evidence from Hong Kong's "Children of 1997" Birth Cohort. A. J. Epidem, 185 (10), 914-923 (2017).

9. McGuinn, L. A. et al. Residential proximity to traffic and female pubertal development. Environ. Int, 94, 635-641 (2016).

10. McConnell, R. et al. A longitudinal cohort study of body mass index and childhood exposure to secondhand tobacco smoke and air pollution, the Southern California Children's Health Study. Environ. Health Perspect, 123 (4), 360-366 (2015).

11. Nikolic, M., Stankovi, A. \& Jovi, S. Effects of air pollution on growth in schoolchildren. Coll. Antropol, 38 (2), 493-497 (2014).

12. Schell, L. M., Denham, M., Stark, A. D., Parsons, P. J. \& Schulte, E. E. Growth of intants' length, weight, head and arm circumference in relation to low levels of blood lead measured serially. Am. J. Hum. Biol, 21 (2), 180-187 (2009).

13. Denham, M. et al. Akwesasne Task Force on the Environmentet al. Relationship of lead, mercury, mirex, dichlorodiphenyldichloroethylene, hexachlorobenzene, and polychlorinated biphenyls to timing of menarche among Akwesasne Mohawk girls., 115, e127-e134 (2005).

14. Wu, T., Buck, G. M. \& Mendola, P. Blood lead levels and sexual maturation in U.S. girls, The Third National Health and Nutrition Examination Survey, 1988-1994. Environ. Health Perspect, 111, 737741 (2003).

15. Gerdhem, P. \& Obrant, K. J. Bone mineral density in old age, the influence of age at menarche and menopause. J. Bone Min. Metab, 22, 372-375 (2004).

16. He, C. et al. Age at menarche and risk of type 2 diabetes, results from 2 large prospective cohort studies. Am. J. Epidemiol, 171 (3), 334-44 (2010).

17. Aksglaede, L., Sorensen, K., Petersen, J. H., Skakkebaek, N. E. \& Juul, A. Recent decline in age at breast development, the Copenhagen Puberty Study., 123, e932-e939 (2009).

18. Gong, T. T., Wu, Q. J., Vogtmann, E., Lin, B. \& Wang, Y. L. Age at menarche and risk of ovarian cancer, a meta-analysis of epidemiological studies. Int. J. Cancer, 132 (12), 2894-900 (2013). 
19. Lakshman, R. et al. Early age at menarche associated with cardiovascular disease and mortality. J. Clin. Endocrinol. Metab, 94 (12), 4953-60 (2000).

20. Harris, M. A., Prior, J. C. \& Koehoorn, M. Age at menarche in the Canadian population, secular trends and relationship to adulthood BMI. J. Adolesc. Health, 43, 548-554 (2008).

21. McDowell, M. A., Brody, D. J. \& Hughes, J. P. Has age at menarche changed? Results from National Health and Nutrition Examination Survey (NHANES) 1999-2004. J. Adoles.. Health, 44, 227-231 (2007).

22. Jung, E. M. et al. Does exposure to PM10 decrease age at menarche? Envir. Int, 117, 16-21 (2018).

23. Brook, R. D. et al. American Heart Association Council on Epidemiology and Prevention, Council on the Kidney in Cardiovascular Disease, and Council on Nutrition, Physical Activity and Metabolism. Particulate matter air pollution and cardiovascular disease, An update to the scientific statement from the American Heart Association., 121 (21), 2331-78 (2010).

24. Rudel, R. A. \& Perovich, L. J. 2009. Endocrine disrupting chemicals in indoor and outdoor air. Atmos. Environ. 43 (1), 170-181 (1994)

25. Brunekreef, B. \& Holgate, S. T. Air pollution and health., 360, 1233-1242 (2002).

26. Lee, J. E., Jung, H. W., Lee, Y. J. \& Lee, Y. A. Early-life exposure to endocrine-disrupting chemicals and pubertal development in girls. Ann Pediatr Endocrinol Metab, 24 (2), 78-91 (2019).

27. Mouritsen, A. et al. Juulet A. Hypothesis, exposure to endocrine-disrupting chemicals may interfere with timing of puberty. Int. J. Androl, 33 (2), 346-359 (2010).

28. De Coster, S. \& van Larebeke, N. Endocrine-disrupting chemicals, associated disorders and mechanisms of action. Environ. Public Health, 201, 713696 (2012).

29. Callen, M. S., de la Cruz, M. T., Lopez, J. M. \& Mastral, A. M. PAH in airborne particulate matter. Carcinogenic character of PM10 samples and assessment of the energy generation impact. Fuel. Proces. Technol, 92, 176-182 (2011).

30. Hombach-Klonisch, S., Pocar, P., Kietz, S. \& Klonisch, T. Molecular actions of polyhalogenated arylhydrocarbons (PAHs) in female reproduction. Curr. Med. Chem, 12, 599-616 (2005).

31. Merklinger-Gruchala, A., Jasienska, G. \& Kapiszewska, M. Effect of air pollution on menstrual cycle length-a prognostic factor of women's reproductive health. In. J. Environ. Res. Pub. Health, 14, 816 (2017).

32. Thiering, E. et al. Associations or residential long-term air pollution exposures and satellite derived greenness with insulin resistance in German adolescents. Environ. Health Perspect, 124 (8), 12911298 (2016).

33. Pedersen, M. et al. Ambient air pollution and low birthweight, a European cohort study (ESCAPE).Lancet Respir. Med.1 (9),695-704 